\title{
Pulmonary Embolism Mimicking Pneumonia, Acute Heart Failure and Myocardial Infarction: A Case Report
}

\author{
Hayatu Umar ${ }^{1}$, Augie Ibrahim ${ }^{1}$, Akintomide F. Akindotun ${ }^{1}$, Muhammed E. Hadiza', \\ Aminu M. Sakajiki ${ }^{1}$, Maiyaki S. Abubakar ${ }^{1}$, Balarabe A. Salisu ${ }^{1}$, Mehmet S. Ulgen ${ }^{2}$, \\ Enver Erbilen², Mustafa Kirman²
}

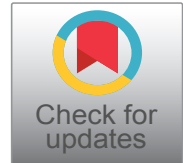

${ }^{1}$ Department of Internal, Usmanu Danfodiyo University Teaching Hospital, Sokoto, Nigeria

${ }^{2}$ Nizamiye Hospital, Abuja, Nigeria

*Corresponding author: Dr. Hayatu Umar, Department of Internal, Usmanu Danfodiyo University Teaching Hospital, Sokoto, Nigeria, Tel: +2348065968412

\begin{abstract}
Introduction: Venous thromboembolism is a common medical condition encountered in emergency departments and is a major contributor to global disease burden. It's often misdiagnosed in critically ill patients despite being common because of its non-specific features, and diagnosis a times is at autopsy. Hence, it's a masquerader that can mimic other cardiorespiratory diseases.

Case presentation: We present a 66-year-old man with pulmonary embolism whose presentation mimicked pneumonia, acute heart failure and myocardial infarction, and a review of diagnostic and treatment challenges in poor resource setting was highlighted.

Conclusion: In poor resource settings with inadequate diagnostic modalities and limited treatment options, pulmonary embolism should be considered in a patient with identifiable risk factors for venous thromboembolism whose admitting provisional diagnosis was Pneumonia or acute left ventricular failure or myocardial infarction who failed to improve with standard therapy.
\end{abstract}

\section{Learning Points}

- Any patient with risk factors for Venous thromboembolism (VTE) and provisional diagnosis of pneumonia or acute heart failure or myocardial infarction, who despite standard management for these clinical conditions failed to improve, a low threshold for pulmonary embolism should be maintained.
- Absence of facilities for pulmonary angiography, Computerized Tomography pulmonary angiography (CTPA) and D-dimer in patient with suspected VTE with large thrombus burden, echocardiography has important diagnostic role in these patients.

- In tertiary health institutions in sub-Saharan Africa with no diagnostic and therapeutic facilities for VTE, if feasible, early referral and collaboration after stabilization with centers having diagnostic facilities and advanced treatment options (intervention/surgical) for VTE may improve the outcome.

\section{Introduction}

Venous thromboembolism (VTE) is a common medical condition encountered in emergency departments and is a major contributor to global disease burden. It's often misdiagnosed in critically ill patients despite being common and diagnosis a times is at autopsy. This disease spectrum may present either as asymptomatic deep venous thrombosis (DVT) or acute pulmonary embolism (PE) leading to death. When established PE occurs without any evidence of DVT, this is known as de-novo pulmonary embolism [1]. As shown by studies in Western Europe, Australia, Southern Latin America and North America, the annual incidence of VTE ranges between 75 and 269 cases per 100,000 individuals [2,3]. 
Although there are no local studies on incidence of VTE in Nigeria and sub-Saharan Africa to the best of our knowledge, however a study in North-Western Nigeria among doctors in a tertiary hospital found that $93.8 \%$ of respondents know the importance of VTE prophylaxis in clinical practice but only $8.7 \%$ admitted to routinely prescribing VTE prophylaxis [4].

In health care facilities of sub-Saharan Africa, emergency room physicians and other practicing physicians have roles to play in the prophylaxis, early diagnosis including atypical presentation, and management of VTE. To achieve this aim, high index of suspicion is required in patients with identifiable risk factors for VTE who present with symptoms and signs suggestive of diagnosis of pneumonia, acute heart failure and myocardial infarction who failed to respond to conventional standard therapy.

\section{Case Presentation}

A 66-year-old known hypertensive, not diabetic who presented to the emergency department (ED) with 7 days history of dry cough, fever, dyspnoea on exertion and easy fatigability, however no associated paroxysmal nocturnal dyspnea (PND), orthopnea, leg and abdominal swelling. Despite feeling unwell, he embarked on a trip of about 12 hours in a car, shortly after arrival he observed worsening of dyspnea and cough necessitating his presentation to ED. Emergency room examination revealed he was tachypneic with respiratory rate of 44 cycles/minute, centrally cyanosed with oxygen saturation of $84 \%$ at room air, subjectively obese, febrile with a temperature of $37.8{ }^{\circ} \mathrm{C}$, not pale, anicteric, bilateral pitting pedal edema which was more marked on the left leg with $2 \mathrm{~cm}$ difference in circumferential diameter over the calf. His pulse rate was $130 /$ minute, regular, normal volume. His blood pressure was $180 / 100 \mathrm{mmHg}$ in sitting position and heart sound were first (S1) and second (S2). There was reduced breath sound over right lung lower zone posteriorly, with coarse crepitations in the left middle and lower zones posteriorly. Abdominal and neurological examinations were unremarkable. Chest X-ray showed homogeneous opacity over the right lower zone and homogeneous opacity obliterating the left costophrenic angle (Figure 1). Electrocardiogram (ECG) showed sinus tachycardia, nonspecific intraventricular conduction block, premature ventricular contraction, normal QTc-398 ms, PR interval-146 ms and normal axis (Figure 2). Full blood count and differential, urea, creatinine and electrolytes and clotting profile were all within normal range.

The initial diagnosis was community acquired pneumonia (CAP) by 3 consecutive attending physicians. He was started on (IV) Augmentin and tab Azithromycin along with oxygen therapy via a high flow mask and was

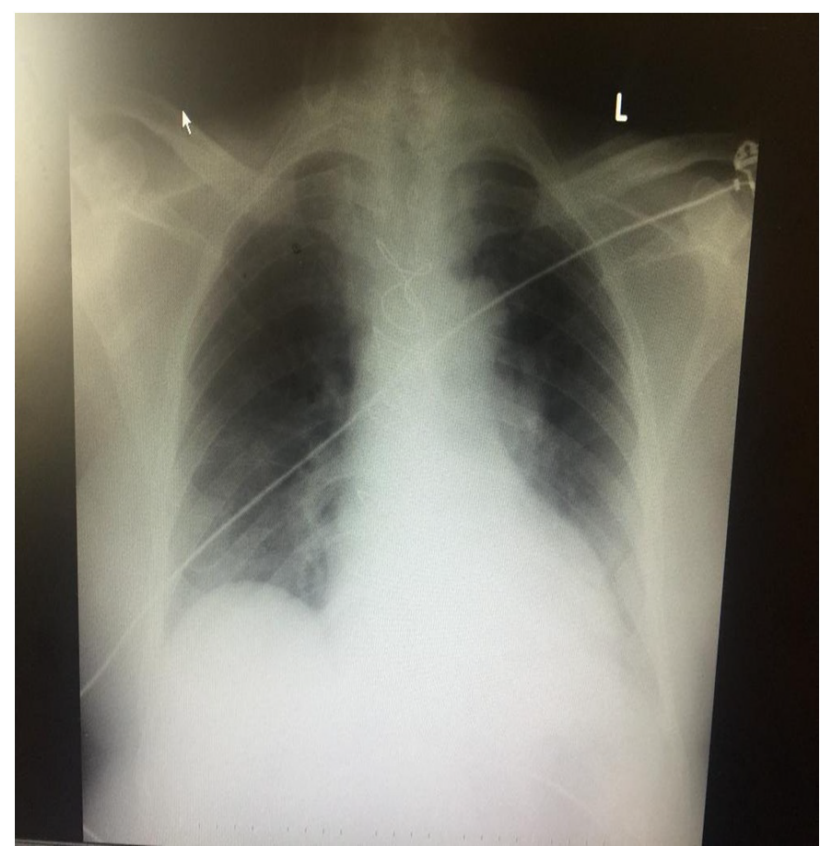

Figure 1: Chest X-ray of index patient showing homogeneous opacity over the right lower zone.

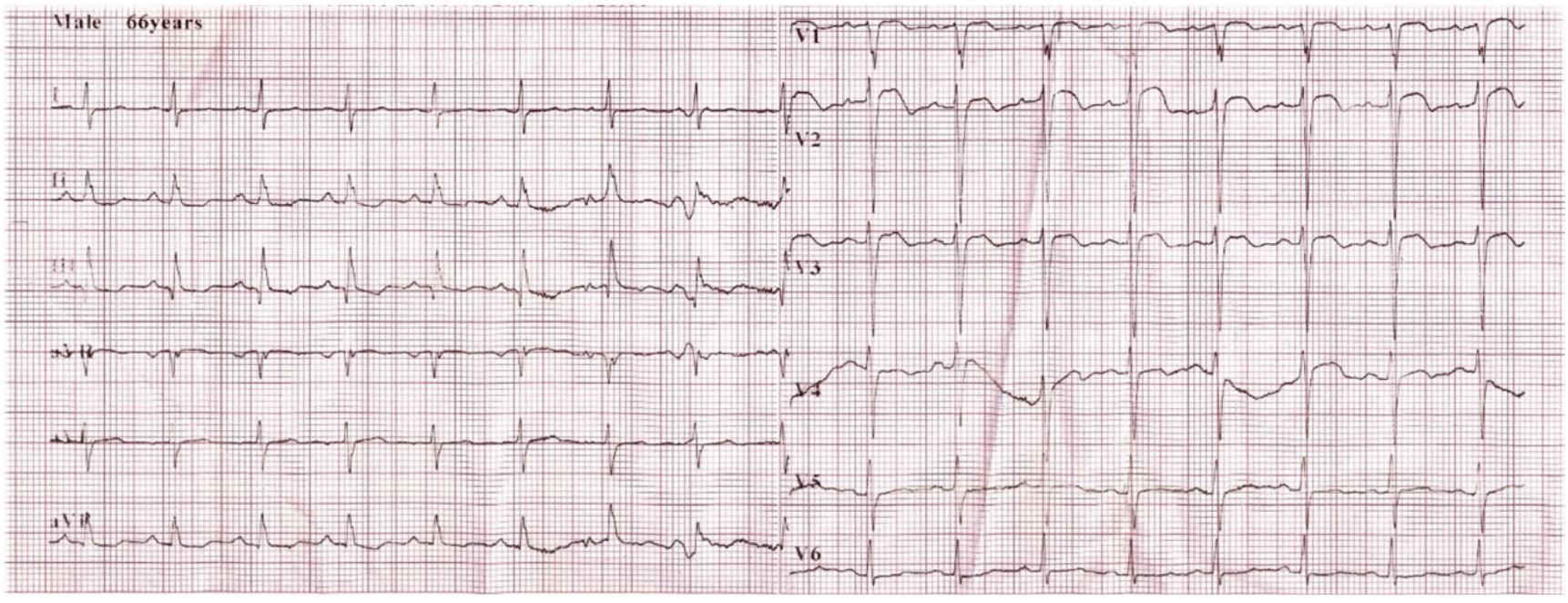

Figure 2: Electrocardiogram (ECG) showing sinus tachycardia, nonspecific intraventricular conduction block and premature ventricular contraction. 


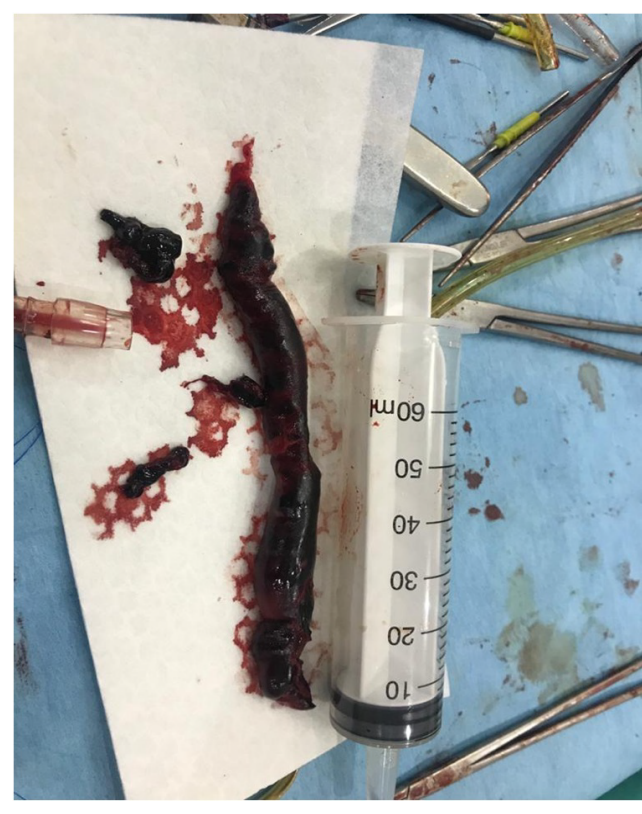

Figure 3: Evacuated right atrial clot post atriotomy.

also started on antihypertensives (Amlodipine, lisinopril). Despite the outlined management, no remarkable improvement was noticed as he was still tachypneic and restless; oxygen saturation was fluctuating between 84 and $90 \%$ at $4 \mathrm{~L} / \mathrm{min}$ of oxygen using oxygen concentrator. Hence, the third attending physician in his follow up review considered hypertensive acute left ventricular failure precipitated by CAP and patient was started on frusemide and spironolactone, however, patient was still severely tachypnoeic. Although arterial blood gas and brain natriuretic peptide were not done because of non-availability. Further review with repeat ECG revealed nonspecific ST-T wave changes, even though there was no associated chest pain, a diagnosis of acute coronary syndrome (ACS) probably myocardial infarction was entertained with a differential diagnosis of venous thromboembolism (VTE). Cardiac markers were within normal range: Troponin I was $0.333 \mathrm{ng} / \mathrm{ml}$ and CK-MB was $9.64 \mathrm{U} / \mathrm{I}$.

Both pulmonologist and cardiologist were invited for review. Echocardiography revealed dilated left atrium (LA: $42.9 \mathrm{~mm}$ ), right atrium (RA: $53.0 \mathrm{~mm}$ ), right ventricle (RVID1: $43.8 \mathrm{~mm}$ ), interventricular septal hypertrophy, normal left ventricular global myocardial wall motion, normal left ventricular systolic function (EF: 75.81\%), multiple mobile right atrial mural thrombus occasionally migrating into right ventricle (Video 1), McConnell's sign was positive (Video 2); regional wall motion abnormality of right basal and mid right ventricular free wall were observed; Severe tricuspid regurgitation (TR) with regurgitant jet area $>10 \mathrm{~cm}^{2}$, mild pulmonary regurgitation (PR), pulmonary arterial hypertension with pulmonary arterial systolic pressure of $44 \mathrm{mmHg}$ at rest. Echocardiographic findings were suggestive of acute pulmonary embolism. Further history taking by the cardiologist revealed past history of road traffic accident with trauma to left leg and right hip joint of 7 years which prompted support of walking stick. Additional physical examination findings of raised jugular venous pressure (JVP) and soft pansystolic murmur at the tricuspid area with tender hepatomegaly were noted. Well's pre-test probability score for DVT and PE were 2 and 7.5 respectively. He was then commenced on subcutaneous low molecular heparin along with warfarin therapy and was transferred to intensive care unit (ICU). IV frusemide and antibiotics were continued and spironolactone stopped. Color duplex scan of lower extremity was requested which was not done as of the time he left to the referral hospital. Pulmonary angiography and CT pulmonary angiography were not available at our center. He was counseled about the gravity of his illness and was also educated on further treatment options, hence he was transferred to another centre where he had open heart surgery: Pulmonary arteriotomy and embolectomy and right atrial clot evacuation (Video 3, Video 4 and Figure 3). Patient is presently doing fine post-surgery.

\section{Discussion}

$\mathrm{PE}$ is a life-threatening medical emergency and is a great masquerader, frequently unrecognized because of its myriad of nonspecific clinical symptoms and its atypical presentation that is often diagnosed at autopsy. The symptoms of PE depend on the thromboembolic burden, the degree of underlying pulmonary parenchymal disease and the ability of the right ventricle to accommodate acute pressure changes [5]. Few case reports were published where PE presented atypically, mimicking pneumonia with symptoms of cough, dyspnea, chest pain and low-grade fever $\left(<39{ }^{\circ} \mathrm{C}\right)$ as in our index case. Other extremely rare presentation includes syncope, shock, ventricular tachycardia and sudden death [6-9].

About half of all patients with acute PE have been recognized to have low to high grade fever at presentation [10-13]. Similarly, pneumonia usually present with fever besides cough, dyspnea, chest pain and occasionally haemoptysis which are common to both PE and pneumonia, hence this can lead to diagnostic dilemma in poor resource settings. However, PE must be considered as a differential diagnosis if patient is not responding to conventional management of pneumonia especially in a patient with risk factors for VTE.

$P E$ and acute left ventricular failure are differential diagnosis of sudden not recent onset dyspnea. In our environment, the commonest cause of acute heart failure is hypertension, therefore the attending physician also thought of hypertensive acute left ventricular failure in our index case, given that the patient is a known hypertensive for more than 10 years, who was not regular on medications, clinic follow-up visit, admitting elevated blood pressure of $180 / 100 \mathrm{mmHg}$, chest examination findings of bilateral coarse crepitations, though in rare instance, coarse crepitations may be observed in heart failure with severe pulmonary edema or coexist- 
ing chest infection. Nevertheless, what may be against hypertensive acute left ventricular failure in our patient is fever of $37.8^{\circ} \mathrm{C}$ and absence of PND, S3 gallop; a hallmark sign of left ventricular dysfunction, radiological evidence of cardiogenic pulmonary oedema, and non-response to standard therapy for heart failure.

Acute myocardial infarction is a differential diagnosis of acute PE. The combination of the lack of pleuritic chest pain, dyspnea, and tachypnea have high specificity (97\%) for absence of PE [14]. Although these 3 cardinal symptoms can be seen in both acute PE and $\mathrm{MI}$, risk factors may be similar. However, characteristic chest pain may differ, as well as location and other associated symptoms such as feeling of impending doom, diaphoresis and Levine's sign may be seen in acute MI with elevated cardiac markers, specific ECG and echocardiographic findings. These features were absent in our index case hence, MI was ruled out.

\section{Conclusion}

In conclusion, many factors may hamper diagnostic and treatment modalities in patients with $\mathrm{PE}$ in poor resource settings like ours. Therefore, any patient with identifiable risk factors for VTE admitted with provisional diagnosis of pneumonia, acute heart failure or myocardial infarction who despite standard therapy for these clinical conditions have not improved, high index of suspicion for PE should be entertained, and focused relevant history taking, meticulous physical examination and relevant case directed investigations may help in establishing a diagnosis of PE and if feasible, early contact and referral to a center with adequate diagnostic and therapeutic options may improve outcome.

\section{Acknowledgement}

The authors would like to thank the patient and his family, all the attending physicians in Usmanu Danfodiyo University Teaching Hospital, Sokoto and Nizamiye Hospital, Abuja for their cooperation and contribution to the successful management of the patient.

\section{Funding}

None.

\section{Conflict of Interests}

None.

\section{Authors Contributions}

All of the authors contributed in planning, conduct and reporting of the work. All contributors are responsible for the overall content as guarantors.

\section{Consent for Publication}

Written informed consent was obtained from the patient to publish this case report. A copy of the written consent is available for review by the editor of this journal.
Source(s) of Financial Support in the Form of Grants (Quote the Number of the grant) Equipment, Drugs

None.

\section{References}

1. Van Gent JM, Zander AL, Olson EJ, Shackford SR, Dunne CE, et al. (2014) Pulmonary embolism without deep venous thrombosis: De novo or missed deep venous thrombosis? J Trauma Acute Care Surg 76: 1270-1274.

2. Konstantinides SV, Barco S, Lankeit M, Meyer G (2016) Management of Pulmonary Embolism: An Update. J Am Coll Cardiol 67: 976-990.

3. Raskob GE, Angchaisuksiri P, Blanco AN, Büller $\mathrm{H}$, Gallus A, et al. (2014) Thrombosis: A Major Contributor to Global Disease Burden. Semin Thromb Hemost 40: 724-735.

4. Makusidi MA, Isah MD, Akintomide F, Abdullahi M, Yakubu A (2016) Knowledge, attitude and practice of venous thromboembolism prophylaxis among medical practitioners in a teaching hospital setting. Orient J Med 28: 36-43.

5. Parsons PE, Wiener-Kronish JP (2012) Critical Care Secrets. ( $5^{\text {th }}$ edn), Elsevier Inc, 1-644.

6. Payus AO, Rajah R, Febriany DC, Mustafa N (2019) Pulmonary embolism masquerading as severe pneumonia: $A$ case report. Open Access Maced J Med Sci 7: 396-399.

7. Saad M, Shaikh DH, Adrish M (2018) A rare case report of a saddle pulmonary embolism presenting with high grade fevers, responsive to anticoagulation. Medicine (Baltimore) 97: e0002.

8. Demircan A, Aygencel G, Keles A, Ozsoylar O, Bildik F (2009) Pulmonary embolism presenting as syncope: A case report. J Med Case Rep 3: 7440.

9. Lucerna A, Espinosa J, Ackley L, Carhart P, Stranges D, et al. (2017) A Case Report on VT from TV: DVT and PE from Prolonged Television Watching 1. Case Reports Pulmonol 2017.

10. Israel HL, Goldstein F (1957) The varied clinical manifestations of pulmonary embolism. Ann Intern Med 47: 202-226.

11. Nucifora G, Badano L, Hysko F, Allocca G, Gianfagna P, et al. (2007) Pulmonary embolism and fever: when should right-sided infective endocarditis be considered? Circulation 115: e173-e176.

12. Murray HW, Ellis GC, Blumenthal DS, Sos TA (1979) Fever and pulmonary thromboembolism. Am J Med 67: 232-235.

13. Swaroop M, Tarbox A (2013) Pulmonary embolism. Int J Crit IIIn Inj Sci 3: 69.

14. Stein PD, Terrin ML, Hales CA, Palevsky HI, Saltzman HA, et al. (1991) Clinical, laboratory, roentgenographic, and electrocardiographic findings in patients with acute pulmonary embolism and no pre-existing cardiac or pulmonary disease. Chest 100: 598-603. 